\title{
Strategic use of obturator prostheses for the rehabilitation of oral cancer patients during the COVID-19 pandemic
}

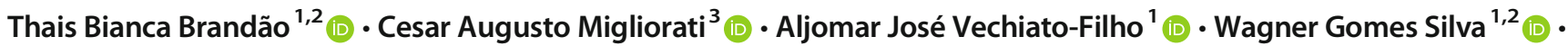 \\ Ana Carolina Prado Ribeiro ${ }^{1,2}$ (D) $\cdot$ Orlando Parise-Junior $^{4} \cdot$ Luiz Paulo Kowalski $^{5,6}$ (D) - Alan Roger Santos-Silva ${ }^{7}$ (D)
}

Received: 22 April 2020 / Accepted: 19 August 2020 / Published online: 28 August 2020

(C) Springer-Verlag GmbH Germany, part of Springer Nature 2020

\begin{abstract}
During the current pandemic scenario, maxillofacial rehabilitation specialists involved with supportive care in cancer must transform its practice to cope with COVID-19 and improve protocols that could quickly return the oral function of complex cancer patients who cannot wait for surgical complex rehabilitation. This includes the role of the maxillofacial prosthodontist for the rehabilitation of surgically treated patients with maxillary cancers by the means of filling obturator prostheses that are considered an optimal scientific-based strategy to reduce hospital stay with excellent pain control, oral function (speech, swallowing, mastication, and facial esthetics), psychologic and quality of life outcomes for the patients following intraoral cancer resection. Therefore, the aim of this commentary was to bring new lights to the strategic use of obturator prostheses for the rehabilitation of oral cancer patients during the COVID-19 pandemic as well as to present a protocol for managing such cases.
\end{abstract}

Keywords COVID-19 $\cdot$ Oral cancer $\cdot$ Rehabilitation $\cdot$ Obturator prosthesis

From the initial identification of the novel coronavirus 2019 disease (COVID-19) passing through the World Health Organization (WHO) recognition as a pandemic to the current chaotic world scenario, specialists discuss criteria for prioritizing surgical treatment for head and neck aggressive malignant tumors [1-3]. Several head and neck surgery services

Thais Bianca Brandão

thais.brandao@hc.fm.usp.br

1 Dental Oncology Service, São Paulo State Cancer Institute (ICESP-FMUSP), Av. Dr. Arnaldo, 251 - Cerqueira César, Sao Paulo 01246-000, Brazil

2 Oral Medicine Department, Hospital Sírio-Libanês, Sao Paulo, Brazil

3 College of Dentistry, University of Florida, Gainesville, FL, USA

4 Head and Neck Surgery Department, Hospital Sírio-Libanês, Sao Paulo, Brazil

5 Head and Neck Surgery Department, AC Camargo Cancer Center, Sao Paulo, Brazil

6 Department of Head and Neck Surgery, University of Sao Paulo Medical School, Sao Paulo, Brazil

7 Oral Diagnosis Department, Piracicaba Dental School, University of Campinas (UNICAMP), Piracicaba, Brazil have prioritized less complex surgical procedures, avoiding longer surgical time for tumor resections and microsurgical reconstructions aiming to reduce the exposure of the patients and team members to the risk of contagion [4]. These decisions were also based on the need for reducing the period of hospitalization and on the fact that microsurgical reconstructions often require long-standing patient monitoring in intensive care units, which may not be currently available due to the pandemic.

In this scenario, when the medical teams consider the surgical procedure unavoidable (weighing the risks of contamination of the patient/team and prognosis of the oncological disease), the multidisciplinary teams must be involved to allow the best possible results. This includes the role of the maxillofacial prosthodontist for the rehabilitation of surgically treated patients with maxillary cancers by the means of filling obturator prostheses that are considered an optimal scientificbased strategy to reduce hospital stay with excellent pain control, reestablishment of oral function (speech, swallowing, mastication, and facial esthetics), and the improvement of psychological and quality of life outcomes for the patients following intraoral cancer resection (Fig. 1) [5-7].

The strategic use of obturator prostheses for the rehabilitation of oral cancer patients has the potential to reduce the professional burden of head and neck surgical oncology staff 


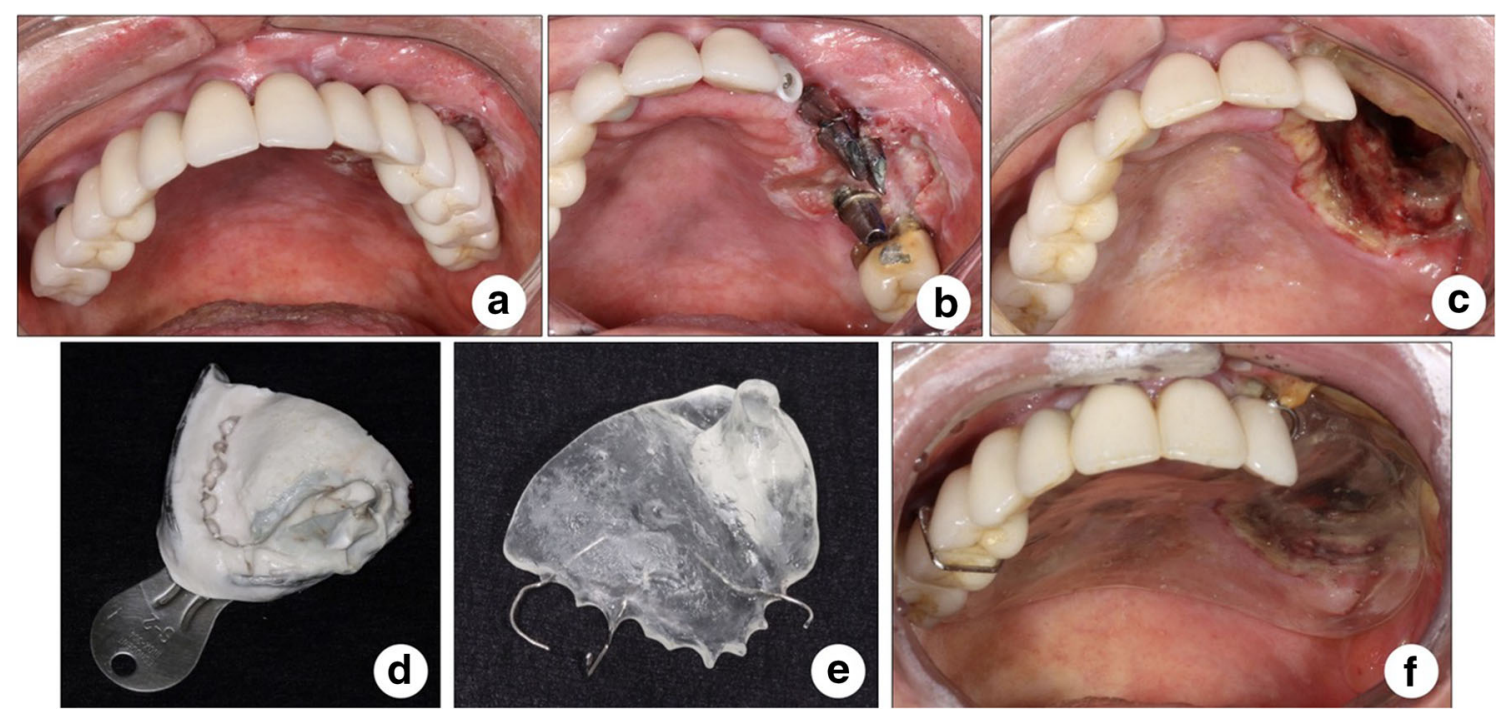

Fig. 1 Step-by-step procedure for fabricating immediate obturator. a Initial clinical aspect. Note the cancer lesion under the prosthetic reconstruction on the left side of maxilla. b Cement-retained multiple implant crowns were removed before partial maxillectomy. c Postoperative aspect 3 days after surgery showing the oronasal communication. Note that the crown of tooth no. 22 was re-cemented before impression. d Conventional impression with irreversible hydrocolloid

member during the COVID-19 pandemic. Potential benefits include a reduction in the number of patients' visits by the surgeons because of improved control of pain and oral infections and decreased risk of bacteremia. Also, the reestablishment of oral function immediately following wide surgical resections will help the patient's nutritional status and weight maintenance. In addition, the protocol allows for a prompt surgical recover and hospital discharge. Our team observed a similar number of obturators for oral cancer patients when comparing this same period of time (March to June) between the years 2019 and 2020, 8 versus 6, respectively; however, the number of microsurgical reconstructions dropped from 7 to 0 cases in this same period of time between 2019 and 2020 . This may be considered an additional evidence for the fact that the use of obturator prostheses for oral cancer patients improves not only the overall quality of life of patients but also the professional workflow of head and neck surgical oncology teams.

In addition, the protocol proposed in this commentary allows for a prompt surgical recovery and hospital discharge. Our team confirmed a similar number of delivered obturators for oral cancer patients between March and June of 2019 and 2020,8 versus 6 , respectively. However, the number of microsurgical reconstructions dropped from 7 to 0 cases in this same period of time. This may be considered an additional evidence that the use of obturator prostheses for oral cancer patients improves not only the overall quality of life of patients but also the professional workflow of the head and neck surgical oncology teams.
(Cavex Colorchange Type 1; Cavex Holland BV, The Netherlands) after the individualization of the stock tray by using polysiloxane impression material (Zetaplus, Zhermack SpA, Italy). e Immediate obturator was fabricated with autopolymerizing acrylic resin and stainless steel clips. f Prosthesis in position. No escape of fluids was observed and speech was restored satisfactorily

In our center, the prosthodontic rehabilitation of oral cancer patients is divided into three phases, as follows:

1. Immediate surgical obturation: impressions by using arbitrary extended trays and altered casts are obtained before surgery, and the prosthesis is placed during surgical procedure and packed by using circum-zygomatic wires, when necessary

2. Delayed surgical obturation: impressions of the defect are generally performed at $\approx 7$ days after surgery and prosthesis is fabricated with an obturator by using a non-altered working cast

3. Definitive obturation: involves definitive prostheses after 3 to 4 months post surgically or 6 to 12 months after radiotherapy

Table 1 and Fig. 2 summarize the impact of the COVID-19 pandemic in the workflow of the fabrication of obturator prostheses for the rehabilitation of oral cancer patients.

Following the guidelines to avoid unnecessary consultations, molding for the fabrication of the surgical obturator prosthesis must be performed at the same time of hospitalization, followed by intraoperative installation. In order to reduce follow-up for necessary adjustments, a reline or an impression and installation of a new prosthesis (interim obturator) must be performed on the day of hospital discharge - reducing the ideal time of this phase transition from 7 days to approximately 3 days [10]. Obviously, this is a desirable outcome during the pandemic of COVID-19. In addition, such procedure will 
Table 1 Differences between traditional versus COVID-19 workflow for obturator prostheses

\begin{tabular}{|c|c|c|}
\hline & $\begin{array}{l}\text { Traditional workflow } \\
(\approx \text { days/local })\end{array}$ & $\begin{array}{l}\text { COVID-19 workflow } \\
(\approx \text { days/local })\end{array}$ \\
\hline $\begin{array}{l}\text { Consultations and molding for surgical obturator prosthesis } \\
\text { fabrication }\end{array}$ & $\begin{array}{l}\text { At least } 7 \text { days before surgery/dental } \\
\text { clinic }\end{array}$ & $\begin{array}{l}1 \text { day before surgery/surgical admission at the } \\
\text { hospital }\end{array}$ \\
\hline Installation of surgical obturator prosthesis & During the surgery/operative room & During the surgery/operative room \\
\hline $\begin{array}{l}\text { New impression and installation of interim obturator } \\
\text { prosthesis }\end{array}$ & 7 days after the surgery/dental clinic & $\begin{array}{l}3 \text { days after the surgery or at the day of hospital } \\
\text { discharge/surgical admission at the hospital }\end{array}$ \\
\hline First control consultation of interim obturator prosthesis & 14 days after the surgery/dental clinic & $\begin{array}{l}10 \text { days after the surgery/video or telephone } \\
\text { calls }\end{array}$ \\
\hline Second control consultation of interim obturator prosthesis & 21 days after the surgery/dental clinic & 23 days after the surgery/dental clinic \\
\hline
\end{tabular}

ensure a longer period of time until the need for a replacement or relining of the prosthesis. Also, with these protective measures, telephone calls, and, mainly, video calls are considered reliable strategies for patients' guidance and functional assessments [11]. When consultation is unavoidable, breaks in appointments are allowed for proper time of environment disinfection between patients. Of importance is the education of the patient to arrive at the scheduled time, minimizing long waiting. Whenever possible, the patient's family member or carer should not enter the dental office.

The COVID-19 pandemic presented time-sensitive challenges and urgent issues that require developing new and rapid solutions as alternatives to routine standard evidence-based protocols which require substantial time and interactions

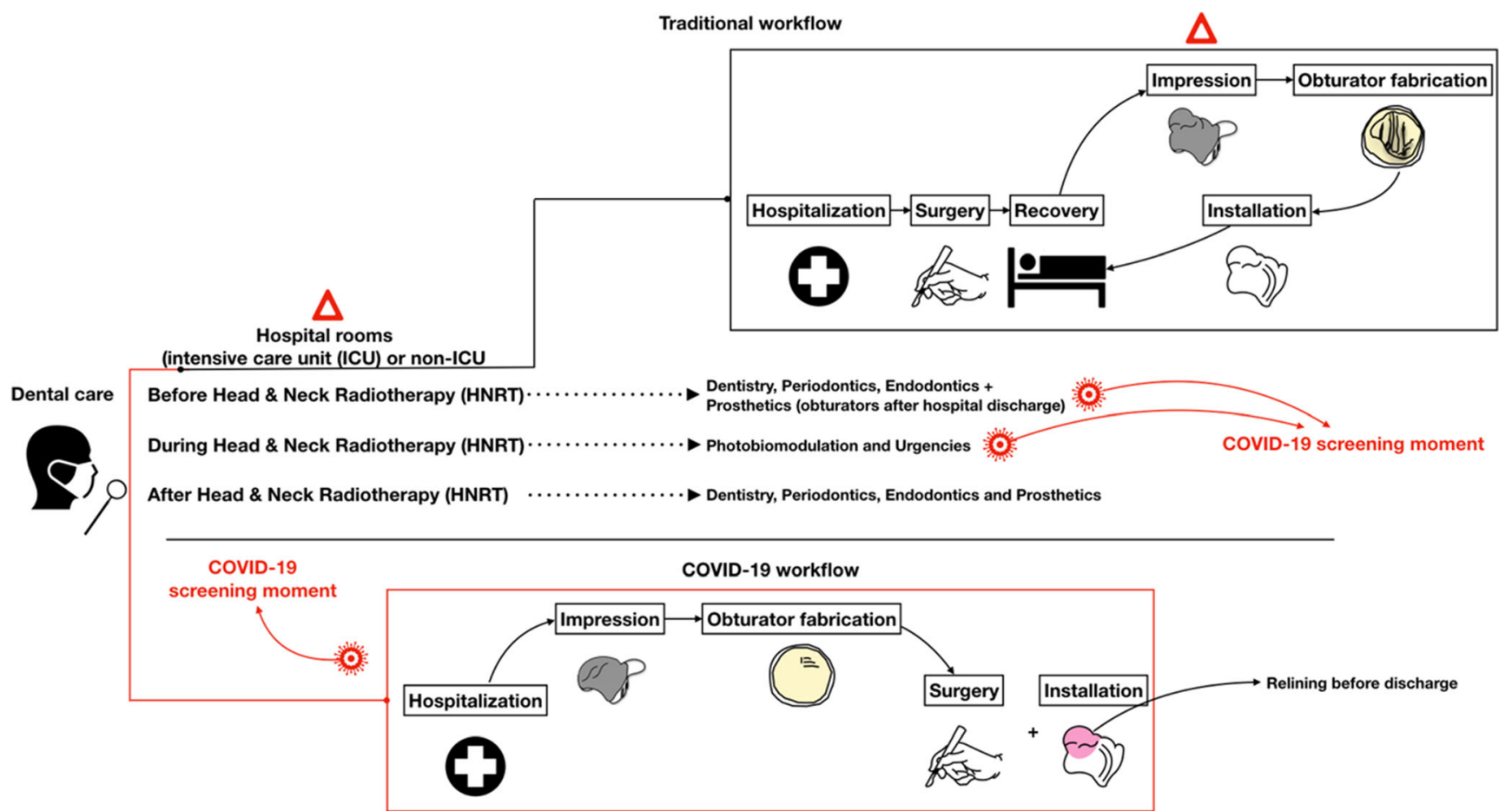

Fig. 2 Traditional versus COVID-19 workflow for obturator prostheses. Note the differences in the sequence for fabricating obturator prostheses between both scenarios. In the COVID-19 pandemic, a symbiosis between phases 1 and 2 of the traditional prosthetic treatment for patients subjected to maxillectomies is suggested whereas the impression will be conducted when the patient is admitted to the hospital for surgery preparation, and prosthesis will be installed at surgical procedure without packing, and it will be relined before patient's discharge. Such workflow will provide a short hospitalization period and delay the return of the patient to dental ambulatory because the bulb part of the obturator prosthesis may improve the restoration of oral functioning (speech and swallowing, for example) for a prolonged period. Besides, this strategy may turn available hospital rooms, reduce PPE intake and the demand for health professionals similar to other rehabilitation approaches [8]. The present figure also illustrates the hospitalization burden of the COVID19 pandemic, in which tertiary hospitals had to implement new strategies of care in order to reduce the PPE intake and hospital resources, support the high demand for IUC beds, and also to prevent COVID-19 dissemination and infection of patients and health professionals [8, 9]. Observe that the proposed workflow for obturator prostheses is not inserted in the well-known situations for COVID-19 contagion $(\Delta)$ and screening moments, reducing the risk of health professional infection 


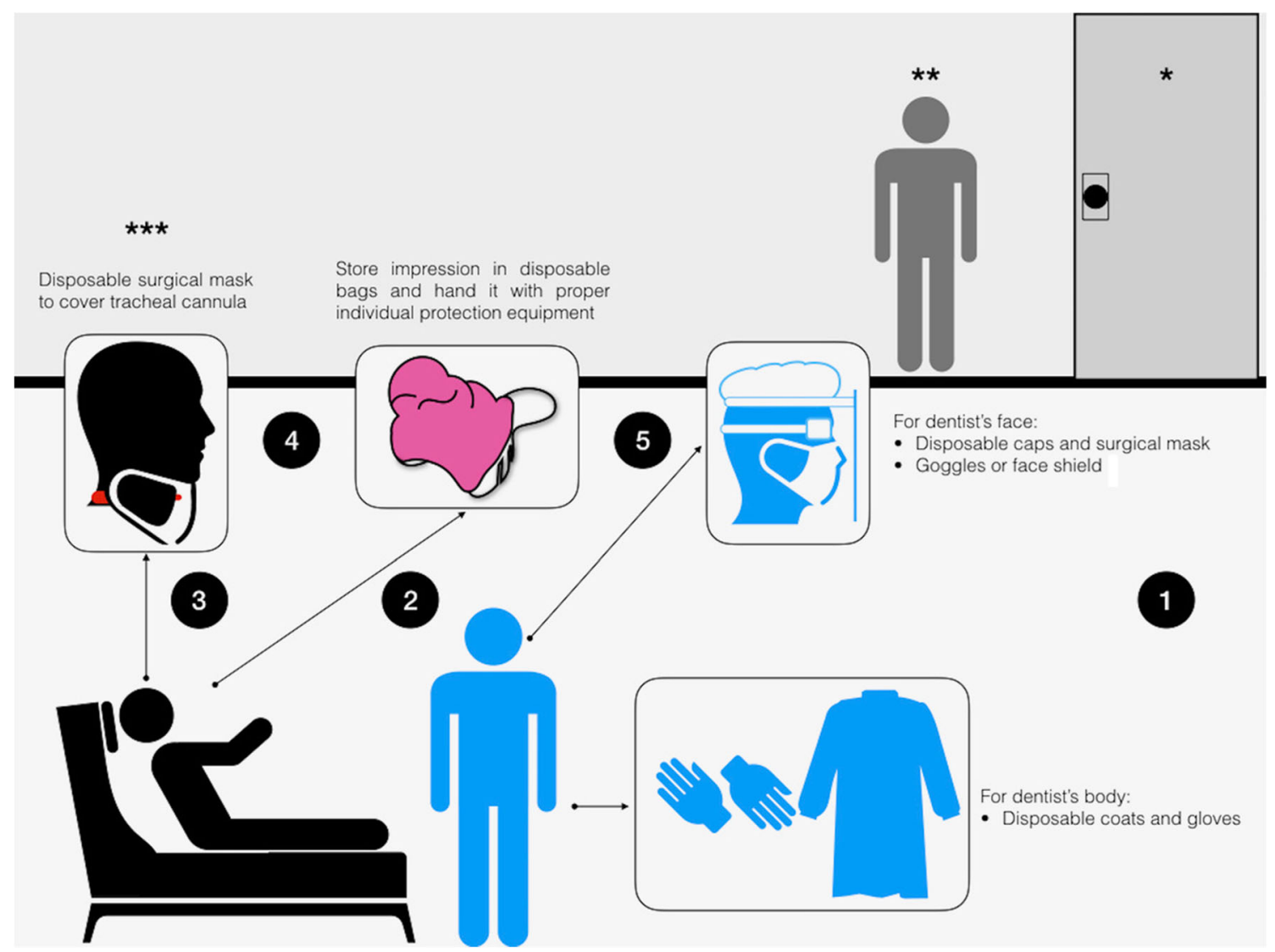

Fig. 3 Scheme summarizing all information presented herein to deliver obturators for patients with maxillectomy defects for three main situations: (1) before surgical procedure (phase 1 of the prosthetic treatment), (2) when dentists are requested after the surgical removal of the tumor and/or after hospital discharge (phase 2). *The door of the hospital room should be closed with low pressure inside in cases of COVID-19 suspicion or confirmation. If a low-pressure hospital room is not possible, one way to provide airflow should be provided (the door or window should be opened), minding to not put other individuals in risk of infection. Otherwise, the procedure should be performed in a better moment. **If possible, the patient's family member or carer should not enter the hospital room. ***Patients with advanced tumor stages may be subjected to neck dissection with protective tracheostomy in complementation to solid tumor removal. They often develop cough and a considerable quantity of secretive content, which can be a significant infection route. Therefore, their tracheal cannula should be covered with a disposable surgical mask. Professionals should sanitize their hands with water and soap or alcohol gel (1) before patient examination, (2) before dental procedures, (3) after touching the patient, (4) after touching equipment without disinfection, and (5) after touching the oral mucosa or body fluids [14].

surgery of complex cancer cases. By strategically altering the prosthodontic rehabilitation protocol, patients can receive necessary cancer care and avoid the need to go through long hospitalization, frequent follow-up visits for obturator adjustments, and still maintain adequate oral function and trauma control [14]. As an integral part of the described protocol, proper COVID-19 professional protective equipment (PPE) use (disposable working cap, isolation gown, goggles or face shield, disposable N95 mask, and disposable gloves, among others) is a must. Hand-washing techniques (water and soap or $\approx 70 \%$ alcohol gel) must be consistent with World Health Organization protocols designed for health care workers [2].

To facilitate the visualization of the protocol described in the commentary, we present a workflow suggestion based on our experience developed in three different cancer care 
reference centers covering from public to private facilities in São Paulo, Brazil (Fig. 3).

Data availability Not applicable.

\section{Compliance with ethical standards}

Conflict of interest The authors declare that they have no conflict of interest.

Code availability Not applicable.

\section{References}

1. Givi B, Schiff BA, Chinn SB, Clayburgh D, Iyer NG, Jalisi S, Moore MG, Nathan CA, Orloff LA, O'Neill JP, Parker N, Zender C, Morris LGT, Davies L (2020) Safety recommendations for evaluation and surgery of the head and neck during the COVID-19 pandemic. JAMA Otolaryngol Head Neck Surg 146:579. https:// doi.org/10.1001/jamaoto.2020.0780

2. Kowalski LP, Sanabria A, Ridge JA, Ng WT, Bree R, Rinaldo A, Takes RP, Mäkitie AA, Carvalho AL, Bradford CR, Paleri V, Hartl DM, Vander Poorten V, Nixon IJ, Piazza C, Lacy PD, Rodrigo JP, Guntinas-Lichius O, Mendenhall WM, D'Cruz A, Lee AWM, Ferlito A (2020) COVID-19 pandemic: effects and evidencebased recommendations for otolaryngology and head and neck surgery practice. Head Neck 42:1259-1267. https://doi.org/10.1002/ hed.26164

3. Cai YC, Wang W, Li C, Zeng DF, Zhou YQ, Sun RH, Jiang H, Guo $\mathrm{H}$, Wang SX, Jiang J (2020) Treating head and neck tumors during the SARS-CoV-2 epidemic, 2019-2020: Sichuan Cancer Hospital. Head Neck 42:1153-1158. https://doi.org/10.1002/hed.26161

4. Yang Y, Soh HY, Cai ZG et al (2020) Experience of diagnosing and managing patients in oral maxillofacial surgery during the prevention and control period of the new coronavirus pneumonia. Chin J Dent Res 23:57-62. https://doi.org/10.3290/j.cjdr.a44339

5. Phasuk K, Haug SP (2018) Maxillofacial prosthetics. Oral Maxillofac Surg Clin North Am 30:487-497. https://doi.org/10. 1016/j.coms.2018.06.009
6. Cao Y, Yu C, Liu W, Miao C, Han B, Yang J, Li L, Li C (2018) Obturators versus flaps after maxillary oncological ablation: a systematic review and best evidence synthesis. Oral Oncol 82:152161. https://doi.org/10.1016/j.oraloncology.2018.05.019

7. Brandão TB, Vechiato Filho AJ, Batista VE et al (2016) Obturator prostheses versus free tissue transfers: a systematic review of the optimal approach to improving the quality of life for patients with maxillary defects. J Prosthet Dent 115:247-53.e4. https://doi.org/ 10.1016/j.prosdent.2015.08.002

8. Smith SR, Jenq G, Claflin T, Magnant C, Haig AJ, Hurvitz E (2020) Proposed workflow for rehabilitation in a field hospital setting during the COVID-19 pandemic. PM R 12:823-828. https:// doi.org/10.1002/pmrj.12405

9. Wong J, Goh QY, Tan Z, Lie SA, Tay YC, Ng SY, Soh CR (2020) Preparing for a COVID-19 pandemic: a review of operating room outbreak response measures in a large tertiary Hospital in Singapore. Can J Anaesth 67:732-745. https://doi.org/10.1007/ s12630-020-01620-9

10. Acharya V, Chambers MS (2015) Maxillofacial prosthodontic rehabilitation of a patient with oral complications during and after multimodality therapy for the management of oral squamous cell carcinoma. J Prosthet Dent 113:651-655. https://doi.org/10.1016/j. prosdent.2014.12.010

11. Ignatius E, Perälä S, Mäkelä K (2010) Use of videoconferencing for consultation in dental prosthetics and oral rehabilitation. J Telemed Telecare 16:467-470. https://doi.org/10.1258/jtt.2010.100303

12. Kampf G, Todt D, Pfaender S, Steinmann E (2020) Persistence of coronaviruses on inanimate surfaces and its inactivation with biocidal agents. J Hosp Infect 104:246-251. https://doi.org/10.1016/j. jhin.2020.01.022

13. Peng X, Xu X, Li Y, Cheng L, Zhou X, Ren B (2020) Transmission routes of 2019-nCoV and controls in dental practice. Int J Oral Sci 12:9. https://doi.org/10.1038/s41368-020-0075-9

14. Kampf G, Todt D, Pfaender S, Steinmann E (2020) Persistence of coronaviruses on inanimate surfaces and their inactivation with biocidal agents. J Hosp Infect 104:246-251. https://doi.org/10.1016/j. jhin.2020.01.022

Publisher's note Springer Nature remains neutral with regard to jurisdictional claims in published maps and institutional affiliations. 